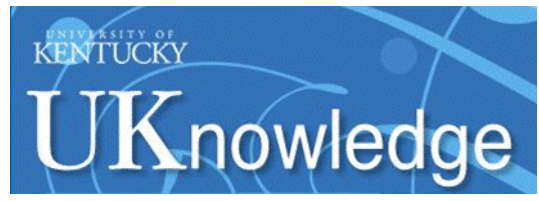

University of Kentucky

UKnowledge

4-1-2002

\title{
Dissipative Heating and Quasar Emission Lines
}

\author{
Mark Bottorff \\ University of Kentucky \\ Gary J. Ferland \\ University of Kentucky, gary@uky.edu
}

Follow this and additional works at: https://uknowledge.uky.edu/physastron_facpub

Part of the Astrophysics and Astronomy Commons, and the Physics Commons

Right click to open a feedback form in a new tab to let us know how this document benefits you.

\section{Repository Citation}

Bottorff, Mark and Ferland, Gary J., "Dissipative Heating and Quasar Emission Lines" (2002). Physics and Astronomy Faculty Publications. 103.

https://uknowledge.uky.edu/physastron_facpub/103

This Article is brought to you for free and open access by the Physics and Astronomy at UKnowledge. It has been accepted for inclusion in Physics and Astronomy Faculty Publications by an authorized administrator of UKnowledge. For more information, please contact UKnowledge@lsv.uky.edu. 


\section{Dissipative Heating and Quasar Emission Lines}

Digital Object Identifier (DOI)

http://dx.doi.org/10.1086/339058

\section{Notes/Citation Information}

Published in The Astrophysical Journal, v. 568, no. 2, p. 581-591.

(c) 2002. The American Astronomical Society. All rights reserved. Printed in the U.S.A.

The copyright holder has granted permission for posting the article here. 


\title{
DISSIPATIVE HEATING AND QUASAR EMISSION LINES
}

\author{
Mark BottorfF and Gary Ferland \\ Department of Physics and Astronomy, 177 Chemistry/Physics Building, University of Kentucky, Lexington, KY 40506 \\ Received 2001 May 21; accepted 2001 December 7
}

\begin{abstract}
Recent observations reveal that the profiles of emission lines of active galactic nuclei are too smooth to be produced by discrete thermal $\left(T \sim 10^{4} \mathrm{~K}\right)$ clouds. The lines may also be too bright to be powered by the continuum unless a large covering factor or additional heating mechanisms are present. We have been investigating one possible explanation of these observations, namely, that the clouds are turbulent. This paper focuses on observational effects caused by dissipation of turbulent energy into cloud heating. We find that internal heating can explain these observations. Clouds energized by both the ionizing continuum and dissipative heating are more efficient line emitters than those powered by the continuum alone. The turbulent velocity field broadens the emission contributions of individual line-emitting clouds so that they overlap, smoothing the line profile. We have broad success in reproducing the observed emission-line spectrum with a turbulent velocity of $\sim 200 \mathrm{~km} \mathrm{~s}^{-1}$, a cloud density of $10^{10} \mathrm{~cm}^{-3}$, and a column density of $10^{22} \mathrm{~cm}^{-2}$. Dissipative turbulence selectively increases intensities of low-ionization lines, making it possible to obtain the "standard" broad-line region line spectrum with a column density $\sim 10$ times smaller than usually assumed. The presence of dissipative heating could explain two long-standing puzzles in quasar emission-line spectra, namely, the smooth line profiles and the energetics of the spectrum.
\end{abstract}

Subject headings: MHD — quasars: emission lines — quasars: general

\section{INTRODUCTION}

This paper is part of a series that examines the effects of turbulence on emission lines in the broad-line region (BLR) of active galactic nuclei (AGNs). Here we investigate the effects of dissipative turbulence on the emission-line spectrum. It is prompted by recent observations revealing that BLR emission-line profiles are so smooth that they challenge conventional models of emission-line regions, in which the observed line width is due to the macroscopic motions of clouds with thermal line widths. In addition, BLR lines may be too bright to be powered by the observed ionizing continuum unless a large covering factor or additional heating mechanisms are invoked.

A nonturbulent cloud, subject only to photoelectric heating from the ionizing continuum, is able to reproduce most of the bright AGN lines if the density of the cloud is $n \sim 10^{10} \mathrm{~cm}^{-3}$ and it is exposed to a typical AGN continuum with an incident flux of ionizing photons of $\Phi \sim 10^{18.5}$ $\mathrm{cm}^{-2} \mathrm{~s}^{-1}$ (Davidson \& Netzer 1979). The column density of such a cloud is not directly measured, but $N \sim 10^{23} \mathrm{~cm}^{-2}$ is needed to reproduce the intensities of low-ionization lines (Kwan \& Krolik 1981). The corresponding length scale associated with a BLR cloud is therefore $L \approx N / n \sim 10^{13}$ $\mathrm{cm}$. We will refer to such clouds as "standard" BLR clouds, and their parameters are listed in Table 1. The computed spectrum (col. [2]) may be compared with observations (cols. [4] and [5]).

The thermal line width of a standard cloud $\left(10 \mathrm{~km} \mathrm{~s}^{-1}\right.$ for $\mathrm{H})$ is $2-3$ orders of magnitude smaller than the velocity width of observed AGN emission lines $\left(10^{4} \mathrm{~km} \mathrm{~s}^{-1}\right.$; Rees 1987; Peterson 1997). The usual interpretation is that the observed line width is due to the bulk motion of many discrete clouds. In this scenario, at sufficiently high spectral resolution, emission lines should break up into a fine spray of spectral components revealing the presence of individual clouds. This does not happen (Arav et al. 1997, 1998;
Dietrich et al. 1999), suggesting that other (perhaps internal) line-broadening agents are at work, or that the picture is fundamentally flawed.

The too smooth line profiles are not the only problem with the standard model. Photoionization models of clouds using standard parameters reproduce most emission-line intensity ratios. There are some problems, however. Lowionization lines originating deep within the neutral part of a cloud (e.g., $\mathrm{Mg}$ II $\lambda 2798$ and $\mathrm{H} \beta \lambda 4861$ ) are generally predicted to be too weak relative to $\operatorname{Ly} \alpha \lambda 1215$, suggesting that an extra internal heating mechanism may be needed to explain the discrepancy (Dumont, Collin-Souffrin, \& Nazarova 1998). In addition, the total luminosity in emission lines may be greater than that available in ionizing radiation unless a large covering factor or additional heating agents are invoked (Korista, Ferland, \& Baldwin 1997b).

In this paper we calculate the emission-line spectrum of BLR clouds subject to both photoionization and dissipative heating. In $\S 2$ we make a few general comments about observed nonthermal motions in nature, turbulence, and nonthermal motions in AGNs. In $\S 3$ an estimate of the volume-heating rate due to turbulent dissipation is scaled to the density and length scales representative of BLR clouds. In $\S 4$ we discuss the resulting emission-line spectrum. A simple model in which the gas density and differential gas covering fraction are powerlaw functions of radius is presented in $\S 5$, and $\S 6$ gives a discussion and a summary.

\section{NONTHERMAL MOTIONS}

\subsection{General Considerations}

Turbulence can be characterized as either dissipative, in which turbulent motions are converted into heat, or nondissipative, in which the motions persist. Dissipative turbulence may be almost inevitable in nature. Numerical 
TABLE 1

Comparison of the Standard and Best-Fit Dissipative Clouds

\begin{tabular}{|c|c|c|c|c|}
\hline \multirow[b]{2}{*}{$\begin{array}{c}\text { PARAMETER OR BLEND/Ly } \alpha \\
\text { (1) }\end{array}$} & \multirow[b]{2}{*}{$\begin{array}{l}\text { STANDARD } \\
\text { (2) }\end{array}$} & \multirow[b]{2}{*}{$\begin{array}{l}\text { DisSIPATIVE } \\
\text { (3) }\end{array}$} & \multicolumn{2}{|c|}{ OBSERVATION } \\
\hline & & & $\begin{array}{c}\text { Zheng et al. (1997) } \\
\text { (4) }\end{array}$ & $\begin{array}{c}\text { Baldwin et al. (1996) } \\
\text { (5) }\end{array}$ \\
\hline $\log n\left(\mathrm{~cm}^{-3}\right)$ & 10.0 & 10.0 & $\ldots$ & $\ldots$ \\
\hline $\log N\left(\mathrm{~cm}^{-3}\right) \ldots$ & 23.0 & 22.0 & $\ldots$ & $\ldots$ \\
\hline 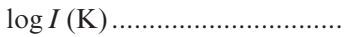 & $\sim 4.0$ & $\sim 4.0$ & $\ldots$ & $\ldots$ \\
\hline 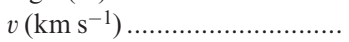 & 0 & 200 & $\ldots$ & $\ldots$ \\
\hline O II $\lambda 834+\mathrm{O}_{\text {III }} \lambda 835 \ldots \ldots \ldots$ & $0.01 \mathrm{y}^{\mathrm{a}}$ & $0.02 \mathrm{y}_{-}$ & 0.014 & $\ldots$ \\
\hline $\mathrm{C}$ III $\lambda 977+\mathrm{L} \gamma \gamma \lambda 835 \ldots \ldots \ldots$ & $0.04 \mathrm{n} \mathrm{y}$ & $0.04 \mathrm{n} \mathrm{y}$ & 0.009 & $0.007-0.20$ \\
\hline 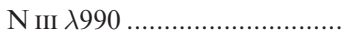 & 0.01 y $n$ & 0.01 y y & 0.011 & 0.013 \\
\hline O II $\lambda 1035+\operatorname{Ly} \beta \lambda 1026 \ldots \ldots$ & $0.02 \mathrm{n} \mathrm{n}^{\mathrm{b}}$ & $0.01 \mathrm{n} \mathrm{n}^{\mathrm{b}}$ & 0.190 & $0.068-0.69$ \\
\hline 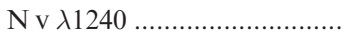 & $0.01 \mathrm{n} \mathrm{n}$ & $0.02 \mathrm{n} \mathrm{n}$ & 0.110 & $0.069-0.99$ \\
\hline Si IV $\lambda 1397+$ O IV $\lambda 1402 \ldots$ & 0.07 y y & 0.07 y y & 0.075 & $0.022-0.50$ \\
\hline 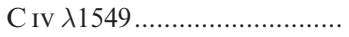 & 0.66 y y & 0.58 y y & 0.620 & $0.087-0.65$ \\
\hline He II $\lambda 1640$ blend $^{c} \ldots \ldots \ldots \ldots \ldots$ & 0.13 y y & 0.11 у у & 0.068 & $0.013-0.14$ \\
\hline C III $\lambda 1909$ blend $^{\mathrm{d}}$.................. & $0.17 \mathrm{y} \mathrm{y}$ & 0.14 y y & 0.163 & $0.076-0.74$ \\
\hline 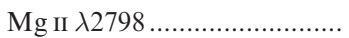 & 0.21 y y & 0.22 y y & 0.250 & $0.15-0.30^{\mathrm{e}}$ \\
\hline 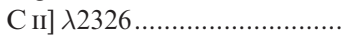 & $0.01 \mathrm{y}$ & $0.02 \mathrm{y}$ & $<0.029^{f}$ & $<0.029^{f}$ \\
\hline 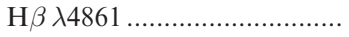 & $0.03 \_n$ & $0.03 \_\mathrm{n}$ & $\ldots$ & $0.07-0.20$ \\
\hline Ly $\alpha / \operatorname{cont} \lambda 1215 \ldots \ldots \ldots \ldots \ldots \ldots$ & 0.89 & 1.07 & 0.076 & $0.03-0.20$ \\
\hline Scorecard .......................... & $7 / 11$ & $7 / 11$ & $7 / 11$ & $8 / 11$ \\
\hline
\end{tabular}

a For Zheng et al. 1997 "y" means that the model line ratio is within a factor of 2 of the mean line profile.

For the Baldwin et al. 1996 data set "y" means that the model line ratio falls within the observed range.

b B00 showed that although a single cloud does not satisfy observations, a blend of clouds does.

c This blend consists of He II $\lambda 1640+\mathrm{O}$ III $\lambda 1663+\mathrm{Al}$ III $\lambda 1671$.

d This blend consists of C III $\lambda 1909+$ Si III $\lambda 1892+$ Al III $\lambda 1859$.

e Ranges are from Baldwin et al. 1995.

f Upper bound from Kwan \& Krolik 1981.

calculations show that even if a gas is taken to be initially uniform in density and temperature, it will develop fully dissipative turbulence when subject to a random velocity field (Porter, Pouquet, \& Woodward 1994). In our own Galaxy, heating due to dissipative turbulence is inferred in diffuse ionized gas (DIG; Minter \& Balser 1997) and in the interstellar medium (ISM).

Dissipative turbulence in the ISM is inferred from observations of molecular lines. These lines have observed widths of a few kilometers per second, making them broader than their thermal widths (a few tenths of a kilometer per second) by $\sim 1$ dex (e.g., Solomon et al. 1987), thus implying supersonic motions. The implied turbulent velocity field is so large that collisions between gas clumps should produce shocks and disassociate the molecules responsible for the observed lines. The molecules persist, however, and in some cases are overabundant in these regions (Lucas \& List 1996). It is hypothesized that the turbulent energy of the gas is rapidly converted into heat via viscous dissipation, thereby providing energy for molecule-creating endothermic reactions. Thus, instead of destroying molecules, the energy may actually help produce them (Falgarone \& Puget 1995).

Turbulence is often accompanied by a magnetic field. Myers \& Goodman (1988) argue that, to within a factor of 2 , there is equipartition between the magnetic energy density $\left(B^{2} / 8 \pi\right)$, the turbulent energy density $\left(\frac{1}{2} \rho v^{2}\right)$, and the gravitational field $(G M \rho / r$; see their Figs. 1 and 2). Crutcher (1999) finds that the magnetic energy and the kinetic energy are approximately equal. This suggests that turbulence in the ISM at large scales is magnetohydrodynamic (MHD) in nature. This is probably also true for the DIG (Minter \& Spangler 1997).

The energy contained in a turbulent gas is distributed in wavenumber covering many decades of physical length scale (Choudhuri 1998). Turbulence phenomenology predicts that the energy in smallest wavenumber modes (the "energy-containing scale") is transferred in a Kolmogorov-type energy cascade through intermediate wavenumber modes (the "inertial range") to the largest wavenumber modes (the "dissipation range"). In the "inertial range" viscosity effects are small, so the cascade proceeds relatively unhindered. The cascade itself is caused by nonlinear interactions between wave modes. As a result the turbulent energy contained in large-scale high-velocity vortices becomes channeled into many small-scale lower velocity vortices. Finally, in the "dissipation range," the cascade is truncated because the Reynolds number is of order unity. Then, viscosity is important and effectively converts turbulent energy into internal heating (Goldstein, Roberts, \& Matthaeus 1995; Choudhuri 1998). Thus, internal volumetric heating will be most pronounced in the smallest structures.

Simulations of turbulence in clouds of the ISM show that MHD turbulence quickly decays unless energy is constantly provided to drive it. Examples of recent numerical simulations include Mac Low (1999), Mac Low, Klesson, \& Burkert (1998), and Stone, Ostriker, \& Gammie (1998). Balsara et al. (1999), using a higher order method, also find similar results, thereby suggesting that the presence of decaying turbulence is robust across numerical schemes. Various energy sources have been suggested as the driver, including 
viscous shear from galactic rotation, gravitational collapse, radiation pressure from nearby ionizing stars, ram pressure from stellar winds and supernovae, and jets from protostars (see related references in Mac Low 1999).

\subsection{Nonthermal Motions in AGN Emission-Line Clouds}

Dissipative turbulence may also be present in AGNs provided that there are physical mechanisms to drive it. AGNs are spatially unresolved (Peterson et al. 1994), so if turbulence exists, it can only be deduced from spectral analysis. Emission lines have widths of $\sim 10^{3}-10^{4} \mathrm{~km} \mathrm{~s}^{-1}$, exceeding the thermal width of the emitting gas by $2-3$ orders of magnitude. This is thought to be due to macroturbulence (e.g., bulk motions of material) rather than microturbulence (e.g., motions on scales smaller than a photon mean free path). AGN emission lines do not indicate that the clouds are predominantly shock heated (Ferland et al. 1996). This is surprising considering that fast flows involving many clouds must occur in the relatively small volume $\left(\sim 1 \mathrm{pc}^{3}\right)$ of the BLR.

The velocity field itself is unknown. Inflow, though not directly observed (see, however, Done \& Krolik 1996), must be present to supply fuel to the AGN central engine. On the other hand, systematic outflows are directly observed with broad blueshifted absorption lines ranging from $\sim 10^{2}$ to $\sim 10^{4} \mathrm{~km} \mathrm{~s}^{-1}$ (Weymann, Carswell, \& Smith 1981; Crenshaw \& Kraemer 1999; Crenshaw et al. 1999; Mathur, Elvis, \& Wilkes 1999).

Various physical scenarios are invoked to explain the broad line profiles, including rotating accretion disks (e.g., Shields 1978; Matthews 1982), continuum-driven winds (Matthews 1986; Chelouche \& Netzer 2001), line-driven winds (e.g., Shlosman, Vitello, \& Shaviv 1985; Murray et al. 1995; Murray \& Chiang 1998; Proga, Stone, \& Kallman 2000), random motions (Kwan \& Carrol 1982), and hydromagnetic motions (Blandford \& Payne 1982; Emmering, Blandford, \& Shlosman 1992; Königle \& Kartje 1994). Each of these scenarios has the capacity to drive dissipative turbulence through mechanisms such as viscous shear, strong velocity gradients, differential or temporal variations of radiation pressure, cloud-to-cloud collisions, and hydrodynamic or hydromagnetic instabilities.

There are reasons to suspect that magnetic fields may be important in the BLR. Rees (1987) suggested that magnetic fields confine BLR clouds. Without some type of confinement mechanism clouds dissipate over a few BLR crossing times. A magnetic field helps contain clouds by constraining their dispersion to directions parallel to the field and may help prevent strong shocks from overheating BLR gas. There are other advantages if a magnetic field is present in BLR gas. It has been shown that a magnetic field stabilizes gas in the inner part of the narrow-line region (NLR) against two-phase thermal instability (Matthews \& Doane 1990) and isobaric perturbations of the total pressure (magnetic pressure plus gas pressure; Bottorff, Korista, \& Shlosman 2000b). Others have shown that dissipative MHD heating has a similar stabilizing influence on the BLR gas itself (Goncalves, Jantenco-Pereira, \& Opher 1996). Finally, the low-density gas that forms the putative intercloud medium of the BLR may provide a significant contribution to line emission if the low-density gas is MHD turbulent and dissipative (Goncalves, Friaca, \& Jantenco-Pereira 2001).
If turbulence (MHD or otherwise) exists in dense $\left(n \sim 10^{10} \mathrm{~cm}^{-3}\right)$ and thick $\left(N \sim 10^{23} \mathrm{~cm}^{-2}\right)$ BLR clouds, then its effects on the transfer of emission lines or continuum will begin to be significant when the velocity dispersion of the turbulence is comparable to the thermal width of the emission lines. For MHD turbulence this occurs when the magnetic pressure is roughly equal to the gas pressure (Rees 1987). The corresponding minimal required magnetic field strength is of the order $\sim 1 \mathrm{G}$ for BLR gas with density $n \sim 10^{10} \mathrm{~cm}^{-3}$ and temperature $T \sim 10^{4} \mathrm{~K}$.

The turbulent velocities are much larger if turbulence is in energy equipartition with gravity. In this case it may have observable effects due to line broadening or clumping of material in the turbulent environment. Bottorff \& Ferland (2000) investigated the effects of large-scale turbulence on the shape of BLR line profiles, showing that smooth line profiles result, and Bottorff et al. (2000a, hereafter B00) investigated the effect of large nondissipative turbulence on emission-line production. Simulations have shown that turbulence gives rise to cloud structures that form and dissipate on short timescales, leaving an overall persistent, clumpy, possibly fractal structure (Elmegreen 1997). In fact, a fractal BLR could explain many features of AGN emission-line spectra (Bottorff \& Ferland 2001).

\section{HEATING BY DISSIPATION OF TURBULENCE}

The structure of dissipative turbulence is very sensitive to the details of the physical processes involved. The physics of turbulence is a rich and complex field, and many competing heating mechanisms are possible (Goldstein et al. 1999). Our goal is to identify the spectral emission line signatures produced by the presence of an extra source of heating acting on a predominantly photoionized cloud. The results we present will mainly be determined by the interplay between this extra heating and the physics of a photoionized gas.

Dimensional arguments suggest that the dissipative power loss per unit volume in a Kolmogorov cascade is

$$
Q=\eta_{v} \rho \frac{v^{3}}{D}\left(\operatorname{ergs~cm}^{-3} \mathrm{~s}^{-1}\right)
$$

In this heating rate, which we adopt, $\eta_{v}$ is a constant, $\rho$ is the mass density of the gas, $v$ is the magnitude of the turbulent velocity field in the cloud, and $D$ is the dissipation length scale. The constant $\eta_{v}$ is of order unity for a wide range of gas pressure and magnetic energy density (Mac Low 1999; Stone et al. 1998).

\subsection{A Simple Cloud Model}

Although the nature of the BLR is uncertain, some information about the ensemble of BLR clouds can be gleaned from the BLR emission lines. This is because they are sensitive to physical conditions within the gas at the smallest scale, including the density and the ionization state of the gas. There is likely to be a considerable range in density, column density, and incident ionizing flux both within any given $\mathrm{AGN}$ and from one $\mathrm{AGN}$ to another. The geometric and kinematic distributions are also unclear. The focus of this paper is on the effects of dissipative turbulence in BLR clouds. We specifically wish to avoid, as much as possible, the dependence of our results on still actively debated scenarios for the global distribution of gas in AGNs. We there- 
fore adopt for analysis a simple one-dimensional slab model with uniform density.

\subsection{The Dissipation Model}

Simulations suggest that turbulence can bring together concentrations of matter for brief periods of time. The idea is that a cloud is a temporary higher density clump of material produced by the confluence of lower density flows (Elmegreen 1997). Modeling shows that any particular cloud (or clump) is a short-lived entity existing for a time of the order of the sound crossing time. As some clouds disperse, others take their place. Thus, if turbulent motions are not too strongly damped, then clouds in a turbulent environment will always be present.

Dissipation will occur primarily in the smaller and denser structures because $Q \propto \rho / D$. Cloud formation and fragmentation cease as energy is diverted into internal heating over the dissipation length. Since the smallest structures in the BLR are presumed to be the BLR clouds, we make the assumption that $D \approx L$. Thus, $Q$ scaled to values representative of BLR clouds becomes

$$
Q \approx 2.3 \times 10^{-3} v_{3}^{3} \frac{n_{10}}{L_{13}} \mathrm{ergs} \mathrm{cm}^{-3} \mathrm{~s}^{-1},
$$

where we have set $\eta_{v} \approx 1.0, \rho \approx 1.4 \times 10^{10} \mu n_{10}$ (where $n_{10}$ is the hydrogen gas particle density in units of $10^{10} \mathrm{~cm}^{-3}, \mu$ is the atomic mass unit, and the factor 1.4 accounts for the relative abundance of $\mathrm{He}$ to $\mathrm{H}$ in the gas, assuming cosmic abundances), $v=v_{3} \times 10^{3} \mathrm{~km} \mathrm{~s}^{-1}\left(\times 10^{5} \mathrm{~cm} \mathrm{~km}^{-1}\right)$, and finally $L=L_{13} \times 10^{13} \mathrm{~cm}$ is the physical scale.

If the energy cascade strictly follows the Kolmogorov scaling law down to structures the size of BLR clouds, then the velocity associated with the smallest vortex structures of size $L$ will be related to the largest structures of size $R$ via $v \approx(L / R)^{1 / 3} V$ (Choudhuri 1998). Assuming that the largest vortices originate from the gravitational interaction of material with the central supermassive black hole, then $V \approx(G M / R)^{1 / 2}$, where $G$ is the universal gravity constant, $M$ is the mass of the central black hole, and $R$ is the radial distance of a BLR cloud from the black hole. In this case the scaling law then becomes

$$
v_{3} \approx 0.025 L_{13}^{1 / 3} R_{18}^{-5 / 6} M_{8}^{1 / 2},
$$

where $R=R_{18} \times 10^{18} \mathrm{~cm}$ and $M=M_{8} \times 10^{8} M_{\odot}$.

We consider the inferred physical parameters for the Seyfert galaxy NGC 5548 to illustrate the order of magnitude of the turbulent velocities expected at the size scale of a BLR cloud. The supermassive black hole in NGC 5548 has an estimated mass of $\sim 7 \times 10^{7} M_{\odot}$, and the C IV-emitting region is $\sim 81$ t-day in radius (Peterson \& Wandel 1999). At a radius of 8 1t-day $V \approx 6700 \mathrm{~km} \mathrm{~s}^{-1}$. By the Kolmogorov scaling law the corresponding local turbulent velocity in individual BLR clouds is (by eq. [3]) $v_{3} \approx 0.5\left(\sim 500 \mathrm{~km} \mathrm{~s}^{-1}\right)$ for $L_{13}=1.0$. In a similar fashion the turbulent velocity in the $\mathrm{H} \beta$-emitting region ( 20 lt-day; Peterson \& Wandel $1999)$ is $v_{3} \approx 0.23\left(\sim 230 \mathrm{~km} \mathrm{~s}^{-1}\right)$. We caution that equation (3) is only a rough scaling. The leading coefficient should therefore be regarded as highly uncertain. We note that the assumption of equipartition in equation (3) is not relevant to the calculations in $\S 4$ where heating in only one cloud is considered. It will, however, be utilized in $\S 5$ when we consider a distribution of clouds.

\section{EMISSION-LINE DIAGNOSTICS}

\subsection{One Central Engine, Two Energy Sources}

Our hypothesis is that BLR clouds intercept energy from both the ionizing continuum and a turbulent velocity field and reprocess these into line emission. The combined effects enhance the heating and cooling of a cloud. Dissipation provides extra heating, which produces an increase in the electron temperature. However, the cooling is also enhanced since the turbulent velocity field decreases the line optical depth of gas by spreading the line opacity over velocity and lowering the line optical depths. The same decrease of optical depth also enhances line pumping by continuum photons since lines can absorb a larger fraction of the continuum as a result of the larger intrinsic line width.

Earlier work on nondissipative turbulence (B00) shows that large values $\left(\sim 10^{3} \mathrm{~km} \mathrm{~s}^{-1}\right)$ can lead to a considerable enhancement (a factor of $\sim 10-1000$ ) in the emission-line luminosity of a BLR cloud just as a result of lowering line optical depths. We expect a similar increase in line luminosity in the case of dissipative turbulence, but with a further increase due to the extra energy provided to the cloud by the additional heating.

The extra energy will predominantly strengthen lower ionization lines since over $90 \%$ of a cloud's mass is in regions where hydrogen is atomic in the standard model. To illustrate, the thickness of the $\mathrm{H}^{+}$region is roughly $\Delta L \approx 10^{23} \Phi / \mathrm{cn}^{2}(\mathrm{~cm})$, where $c$ is the speed of light (Ferland 1999). For $n=10^{10} \mathrm{~cm}^{-3}$ and $\Phi=10^{18.5} \mathrm{~cm}^{-2} \mathrm{~s}^{-1}$ the thickness of the ionized layer is $\Delta L \approx 10^{11} \mathrm{~cm}$. This is only $1 \%$ of the total thickness $\left(L \sim 10^{13} \mathrm{~cm}\right.$ ), so the ratio of the luminosities in the neutral to the ionized regions is $\approx 100$. Lowionization lines originate in this region, where photoelectric heating is weakest, so turbulence should have the greatest additional effect.

The two brightest low-ionization lines are $\mathrm{Mg}$ II $\lambda 2798$ and $\mathrm{H} \beta \lambda 4861$. Previous investigations of the $\mathrm{H} \beta / \mathrm{Ly} \alpha$ ratio (Dumont et al. 1998; Netzer et al. 1995; Netzer 1985; CollinSouffrin 1986) find that this ratio is consistently underpredicted for models that have thermal widths only. When models produce $\mathrm{Mg}$ II $\lambda 2798$ within observational ranges, $\mathrm{H} \beta \lambda 4861$ is underpredicted by a factor $\sim 3-5$. Kaspi \& Netzer (1999) argue that this is a deficiency in today's numerical simulations. We therefore focus on solutions involving dissipative turbulence in which $\mathrm{Mg}$ II $\lambda 2798$ and higher ionization lines are in compliance with observations.

\subsection{Photoionization Calculations}

Here we calculate the emission-line spectrum of a BLR cloud subject to both an external AGN continuum and internal heating by dissipation of turbulence. Our calculations are carried out with the parameters described in $\S 3.1$ and listed in Table 1 . The parameters we vary are the internal turbulence and the column density of the cloud. Solar abundances are assumed.

The effect of dissipative turbulence is included through both energy dissipation and line broadening. Turbulent energy dissipation is included in the calculations by adding equation (2) as an additional heating term to the net heating/cooling balance in the cloud. The turbulent line broadening is included in the calculations by decreasing the line opacity by increasing the Doppler width of the lines. Without turbulence the Doppler width is $V_{\text {Doppler }}=V_{\text {th }}$, where 
$V_{\mathrm{th}}=(2 \mathrm{kT} / \mathrm{m})^{1 / 2}$ is the thermal width of the species, $k$ is Boltzmann's constant, $T$ is the electron temperature, and $m$ is the mass of the ion that produces the line. If dissipative turbulence is included, the Doppler width becomes

$$
V_{\text {Doppler }}=\sqrt{V_{\mathrm{th}}^{2}+v^{2}},
$$

where $v$ is the turbulent velocity. The total widths of emission lines in AGNs are several orders of magnitude larger than the thermal velocity. If even a small part of this is due to turbulence, then the optical depth will change dramatically and alter the line and continuum transfer through the cloud.

The cloud is exposed to a quasar continuum normalized so that the hydrogen-ionizing number flux $\Phi=10^{18.5} \mathrm{~cm}^{-2}$ $\mathrm{s}^{-1}$ at the illuminated face of the cloud (Davidson \& Netzer 1979). The continuum shape is given by

$$
f_{\nu}=\nu^{\alpha_{\mathrm{UV}}} \exp \left(-\frac{h \nu}{k T_{\mathrm{BB}}}\right) \exp \left(-\frac{k T_{\mathrm{IR}}}{h \nu}\right)+a \nu^{\alpha_{\mathrm{X}}},
$$

where $a$ is chosen so that

$$
\frac{f_{\nu}(2 \mathrm{keV})}{f_{\nu}(2500 \AA)}=403.3^{\alpha_{0 x}},
$$

$\alpha_{\mathrm{UV}}=-0.50, T_{\mathrm{BB}}=10^{6.0} \mathrm{~K}, k T_{\mathrm{IR}}=0.01 \mathrm{ryd}, \alpha_{\mathrm{X}}=-1.0$, and $\alpha_{\mathrm{ox}}=-1.40$. The full spectrum between $912.02 \mathrm{~cm}$ and $100.01 \mathrm{MeV}$ is considered. Calculations of the emergent spectrum are carried out using CLOUDY version 96.00.

\subsection{The Emission Lines in a Standard Column Density Cloud}

Figure 1 shows the calculated total line intensities (emergent from both the illuminated and shielded sides of the cloud), relative to Ly $\alpha$, for 12 emission lines and emissionline blends. These are shown as a function of the turbulent velocity $v$. The last panel in the figure shows the strength of Ly $\alpha$ relative to the continuum at $1215 \AA$. The line-tocontinuum ratio is important because it reflects how much energy comes out in lines relative to the ionizing continuum.

In each panel solid horizontal lines show the observed line blend ratios derived from a composite spectrum of quasars. The composite spectrum is from Zheng et al. (1997) and has high signal-to-noise ratio, but it has the drawback that it includes many quasars with broad emission lines, so that the weak lines are either washed out by the continuum or blended with other emission or absorption features. Grayshaded regions bounded by the dashed horizontal lines indicate the range of the line blend ratio measured in a few particularly well observed objects (Baldwin et al. 1996). The individual objects in Baldwin et al. (1996) were selected for their narrower emission lines, which improve the detection limit for weak lines, but this means that these may be atypical objects.

In the range $0<v<80 \mathrm{~km} \mathrm{~s}^{-1}$, six out of 10 line (or line blend) ratios relative to $\operatorname{Ly} \alpha$ simultaneously satisfy the observational constraints of Baldwin et al. (1996), and five out of 10 line ratios satisfy (to within a factor of 2) the observational constraints of Zheng et al. (1997). The intensities of the low-ionization lines $\mathrm{Mg}$ II $\lambda 2798$ and $\mathrm{C}_{\text {II }} \lambda 2326$ become too strong for $v>80-90 \mathrm{~km} \mathrm{~s}^{-1}$. We shall come back to this below, where we argue that clouds may have a column density that is smaller than the value assumed here.

Five line blends, C III $\lambda 977+\operatorname{Ly} \gamma \lambda 973$, Si IV $\lambda 1397$ $+\mathrm{O}$ IV] $\lambda 1402, \mathrm{C}$ IV $\lambda 1549$, He II $\lambda 1640+\mathrm{O}$ III $] \lambda 1663$ $+\mathrm{Al}$ II $\lambda 1671, \quad$ and $\mathrm{C}$ III] $\lambda 1909+\mathrm{Si}$ III] $\lambda 1892+\mathrm{Al}$ III $\lambda 1859$, remain within the Baldwin et al. (1996) range for $80 \leq v \leq 400 \mathrm{~km} \mathrm{~s}^{-1}$. These are relatively insensitive to turbulence over this range. All lines have intensities within a factor of $\sim 2$ of the mean quasar spectrum of Zheng et al. (1997), with the single exception of C III $\lambda 977+\operatorname{Ly} \gamma \lambda 973$, which is a factor of $\sim 4$ too strong.

The lines $\mathrm{He}$ II $\lambda 1640+\mathrm{O}$ III $] \lambda 1663+\mathrm{Al}_{\text {II }} \lambda 1671$ and C III] $\lambda 1909+\mathrm{Si}$ III $\lambda 1892+\mathrm{Al}$ III $\lambda 1859$ stray beyond the observational bounds for $v>400 \mathrm{~km} \mathrm{~s}^{-1}$. The $\mathrm{Al}$ II $\lambda 1671$ and Al III $\lambda 1859$ lines become selectively enhanced with increasing turbulence as a result of diminishing line optical depth (see B00). This is an important constraint because strong Al II $\lambda 1671$ and Al III $\lambda 1859$ are not generally observed. Thus, dissipative turbulence $v>400 \mathrm{~km} \mathrm{~s}^{-1}$ is ruled out in this single-cloud model.

Three ratios are underpredicted. The nitrogen lines N III $\lambda 990$ and $\mathrm{N} v \lambda 1240$ are underpredicted by a factor of $\sim 4$ for $v<400 \mathrm{~km} \mathrm{~s}^{-1}$. We have argued elsewhere that the great intensity of nitrogen lines in AGNs is due to enhanced quasar metallicity (see Korista, Baldwin, \& Ferland 1998 and the review by Hamann \& Ferland 1999). The blend $\mathrm{O}$ VI $\lambda 1034+\operatorname{Ly} \beta \lambda 1026$ is underpredicted for the "typical" BLR cloud at all levels of turbulence, including $0 \mathrm{~km}$ $\mathrm{s}^{-1}$. This is because we are considering a single cloud as representative of the entire BLR. More realistic models include a broad mix of clouds and have greater success (Baldwin et al. 1995). Our earlier efforts invoking globally distributed turbulent behavior (B00; Bottorff \& Ferland 2001) show that this line ratio can indeed be reproduced with a mixture of clouds. In principle, then, we include $\mathrm{O}$ IV as a line consistent with a turbulent environment though the turbulence need not be dissipative.

The $\mathrm{Mg}$ II $\lambda 2798$ and $\mathrm{H} \beta \lambda 4861$ lines, which form in the predominant neutral portion of the cloud, are very sensitive to dissipative turbulence in the range $80<v<400 \mathrm{~km} \mathrm{~s}^{-1}$. This result is significantly different from the nondissipative case. For example, without dissipation, at $v=200 \mathrm{~km} \mathrm{~s}^{-1}$, the relative intensity of $\mathrm{Mg}$ II $\lambda 2798$ is similar to the $v=0$ $\mathrm{km} \mathrm{s}^{-1}$ case and $\mathrm{H} \beta \lambda 4861$ is actually weaker than the $v=0$ $\mathrm{km} \mathrm{s}^{-1}$ case (see Fig. 1 of B00). The reason for the dramatic jump of the primarily collisionally excited Mg II $\lambda 2798$ line is that dissipative heating increases by a factor of $\left(10^{0.5}\right)^{3} \approx 30$ between $\sim 10^{2.0}$ and $\sim 10^{2.5} \mathrm{~km} \mathrm{~s}^{-1}$. Most of the mass of a cloud with a column density of $10^{23} \mathrm{~cm}^{-2}$ lies in neutral material, so this extra heating mainly enhances lowionization species.

The last panel of Figure 1 shows the $\operatorname{Ly} \alpha$ flux relative to the continuum at $1215 \AA$. This figure assumes that the clouds fully cover the continuum source, so comparison of calculation to observation yields an estimate of the total cloud covering factor. The emission-line spectrum increases in brightness relative to the continuum for larger values of turbulence. This means that a smaller covering factor would be required to reproduce observations. A small covering factor, ranging from $\sim 0.01$ (at $v \approx 1000 \mathrm{~km} \mathrm{~s}^{-1}$ ) to $\sim 0.10$ (at $v \approx 10 \mathrm{~km} \mathrm{~s}^{-1}$ ), is needed to reproduce the Zheng et al. (1997) value of Ly $\alpha / 1215 \AA=0.076$. The implied covering factor is $\sim 0.07$ for $v \approx 200 \mathrm{~km} \mathrm{~s}^{-1}$. 

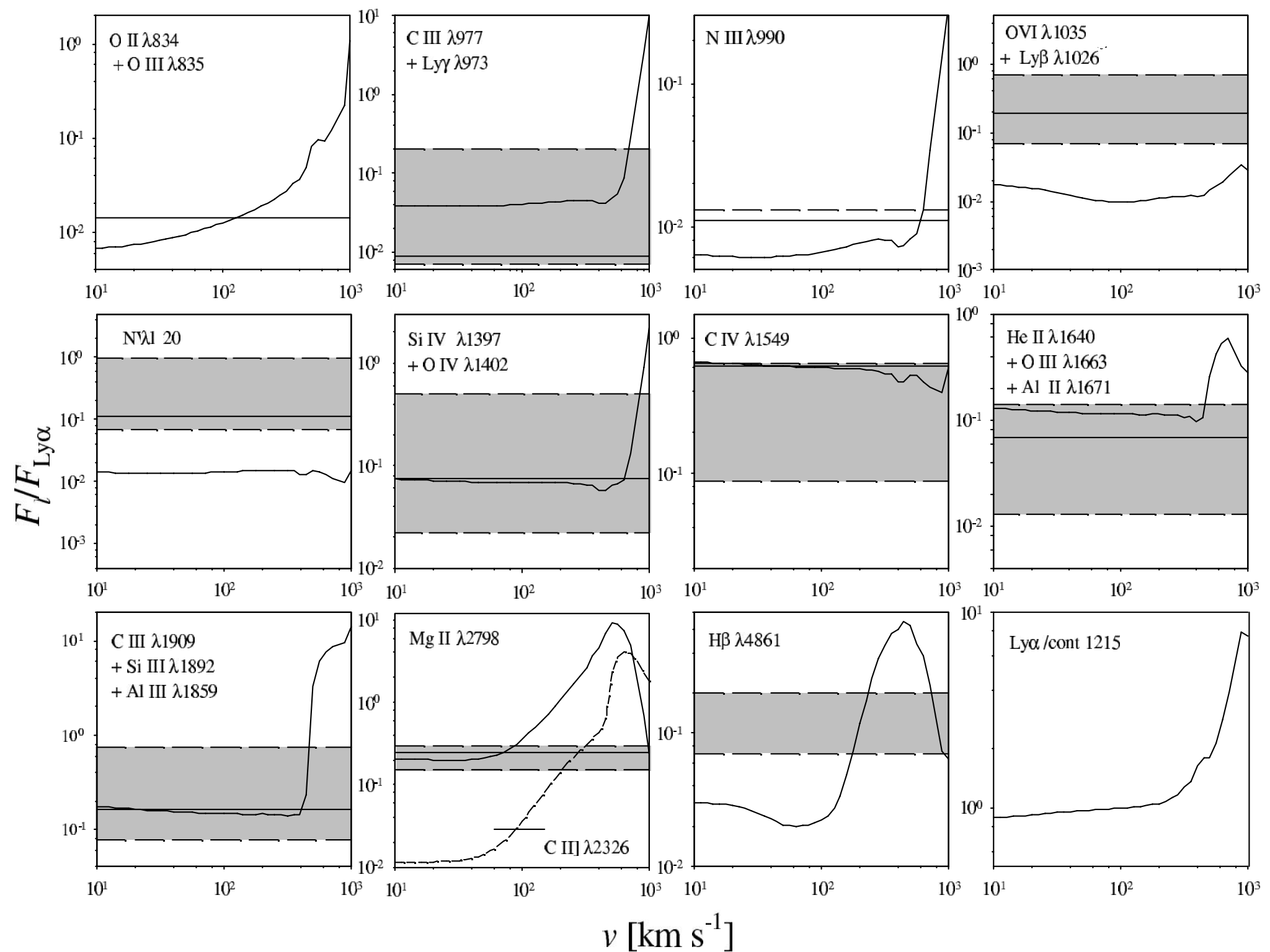

FIG. 1. - Lines and line blend ratios relative to Ly $\alpha$ as a function of turbulent velocity $v$ inside the cloud in $\mathrm{km} \mathrm{s}^{-1}$. Horizontal solid lines show values derived from the mean quasar spectrum of Zheng et al

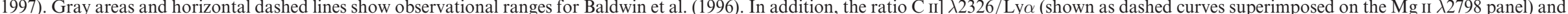
the $\log$ of the ratio of $\mathrm{Ly} \alpha$ relative to the continuum at $1216 \mathrm{~A}(\mathrm{Ly} \alpha / \lambda 1216)$ are shown. 


\subsection{The Emission-Line Spectrum and the Column Density}

The standard cloud column density used above was chosen to reproduce low-ionization lines like $\mathrm{Mg}$ II $\lambda 2798$ and C II] $\lambda 2326$ without turbulence. The previous section shows that these lines are too strong when significant turbulence is present, suggesting that a smaller column density is indicated. Here we vary the column density of a turbulent cloud to deduce a self-consistent column density. In these calculations the cloud parameters and the ionizing flux are kept identical to those for the standard BLR cloud with the exception that the total thickness $L$ (and hence the cloud column density) is varied.

Figure 2 shows the line blends, relative to $\operatorname{Ly} \alpha$, as a function of $\log N$, where $N$ is the column density $\left(\mathrm{cm}^{-2}\right)$. The thin curves show clouds with $v=200 \mathrm{~km} \mathrm{~s}^{-1}$, and the thick curves show $v=400 \mathrm{~km} \mathrm{~s}^{-1}$. The choice $v=400 \mathrm{~km} \mathrm{~s}^{-1}$ is an upper bound because several lines ( $\S 4.3)$ prohibit larger values. Horizontal lines and gray-shaded areas show the same observational ranges as in Figure 1.

As expected, the tightest constraints on the column density come from the low-ionization lines $\mathrm{Mg}$ II $\lambda 2798$ and $\mathrm{C}$ II] $\lambda 2326$, which were the lines used to obtain the standard value (Kwan \& Krolik 1981). C II] $\lambda 2326$ is shown as the dashed curve superimposed on the plot of $\mathrm{Mg}$ II $\lambda 2798$ in Figure 2. Both the low-ionization lines $\mathrm{Mg}$ II $\lambda 2798$ and C II] $\lambda 2326$ and the high-ionization lines agree with observations with a turbulent velocity of $v \approx 200 \mathrm{~km} \mathrm{~s}^{-1}$ and a cloud column density of $\log N \approx 22.0$, a factor of 10 smaller than the standard value. Full predictions of the spectrum of the dissipative cloud with these new parameters are given in Table 1 and discussed in $\S 6$.

\section{TOTAL DISSIPATIVE POWER}

Previous work has suggested that the lines may radiate more power than is present in the ionizing continuum. Basically, a too large covering factor of $0.20-0.40$ is needed for some objects (Korista et al. 1997a). In this section we compute a simple spherically symmetric model of the BLR to show that dissipative heating can provide the extra energy.

\subsection{A Simple BLR Model}

The BLR is a highly stratified environment with higher ionization gas lying closer to the central source. Low-ionization lines are narrow compared to high-ionization lines, and low-ionization lines have smaller intrinsic peak velocities than high-ionization lines (Gaskell 1982; Wilkes 1984; Collin-Souffrin \& Lasota 1988; Espey et al. 1989; Corbin 1990). In addition, reverberation mapping reveals that lowionization lines originate at greater distance from the continuum source than high-ionization lines (Peterson 1993). Since low-ionization lines originate at greater $R$, the average ionization parameter $U=\Phi / n c$ (where $\Phi$ is the number flux of hydrogen-ionizing photons, $n$ is the density, and $c$ is the speed of light) must be a decreasing function of $R$ (i.e., $d U / d R \leq 0)$. Thus,

$$
n_{10} \approx\left(\frac{R_{18}}{R_{18,10}}\right)^{\gamma}
$$

where $R_{18,10}$ normalizes $n_{10}$ and we must have $-2 \leq \gamma$ to guarantee that $U$ decreases with $R$. On the other hand, if the central engine is the source that is driving turbulence, then denser structures will lie at smaller radii since turbulence is expected to be greater there (Bottorff \& Ferland 2001; Elmegreen 1997). Thus, $-2<\gamma<0$. We note that equation (7) should only be taken as representative of the general trend of density with radius. High-turbulence environments will have a variety of densities coexisting in the same region (Elmegreen 1997) with line emission, at any given radius, originating mainly from the clouds that emit most strongly there (Baldwin et al. 1995).

The value of $R_{18,10}$ is chosen by setting $n_{10}=1.0$ when $\Phi=10^{18.5} \mathrm{~cm}^{-1} \mathrm{~s}^{-1}$ (Davidson \& Netzer 1979). The hydrogen-ionizing flux density $\Phi$ as a function of radius is obtained from the bolometric luminosity $L_{b}$ via $\Phi=L_{b} f_{\mathrm{H}} / 4 \pi R^{2}\langle E\rangle$, where $f_{\mathrm{H}}$ is the fraction of the luminosity above 1 ryd and $\langle E\rangle$ is the mean energy per photon above $1 \mathrm{ryd}$. For this example we use $f_{\mathrm{H}} \approx 0.658$ and $\langle E\rangle \approx 44.23$ $\mathrm{eV}$, which corresponds to the parameter choices of equations (5) and (6). If we let $L_{b} \approx \eta L_{\mathrm{Edd}}$, where $\eta$ (not to be confused with $\eta_{v}$ in eq. [1]) is the portion of the accretion efficiency that drives the continuum and $L_{\text {Edd }}$ is the Eddington luminosity (Frank, King, \& Raine 1997), we obtain $\Phi \approx 9.6 \times 10^{17} M_{8} \eta_{-1} / R_{18^{2}} \mathrm{~cm}^{-2} \mathrm{~s}^{-1}$, where $\eta_{-1}=\eta / 0.1$. Setting $\Phi \approx 10^{18.5} \mathrm{~cm}^{-2} \mathrm{~s}^{-1}$ and solving for $R_{18}$ gives

$$
R_{18,10} \approx 0.55\left(M_{8} \eta_{-1}\right)^{1 / 2} .
$$

In addition to letting density vary with radius, we also let the filling factor $\epsilon$ vary with radius as a simple power law. Thus,

$$
\varepsilon_{-6} \approx \varepsilon_{-6,0} R_{18}^{\delta}
$$

where we set $\varepsilon=\varepsilon_{-6} \times 10^{-6}$ since the filling factor, estimated from observation, is of the order $10^{-6}$ (Peterson 1993).

If the cloud size is fixed and we can neglect cloud shadowing, then the differential covering fraction varies with radius as a power law as well since

$$
\varepsilon=n_{c} V_{c}=\left(n_{c} f_{c} A_{c}\right) L=f_{c} \frac{d C}{d R} L
$$

where $n_{c}$ is the number density of BLR clouds per unit volume, $V_{c}=f_{c} A_{c} L$ is the volume of a single BLR cloud with $A_{c}$ the cross-sectional area of the cloud, and the geometric factor $f_{c}$ is of order unity (e.g., for a sphere $f_{c}=\frac{2}{3}$, for an edge-on cylinder of arbitrary cross section $f_{c}=1$ ). For simplicity we assume spherical clouds of diameter $L_{13} \approx 1.0$.

The total covering fraction is estimated to be $C \sim 0.10$ based on the total fraction of sources that have line-of-sight absorption (Weymann 1997). We therefore set the normalization constant, $\varepsilon_{-6,0}$, such that the integrated covering fraction is equal to 0.1 . The total dissipative luminosity is then

$$
\begin{aligned}
P_{\mathrm{BLR}} \approx & \int_{R_{\mathrm{in}}}^{R_{\mathrm{BLR}}} Q \varepsilon 4 \pi R^{2} d R \\
= & \frac{4.5 \times 10^{41} M_{8}^{3 / 2} \varepsilon_{-6,0}}{R_{18,10}^{\gamma}} \\
& \times \int_{R_{18, \mathrm{in}}}^{R_{18, \mathrm{BLR}}} R_{18}^{\gamma+\delta-1 / 2} d R_{18}\left(\mathrm{ergs} \mathrm{s}^{-1}\right) .
\end{aligned}
$$

Next we fix the limits of integration $R_{\mathrm{in}}$ and $R_{\mathrm{BLR}}$. Since our focus is the BLR, we set the inner radius based on the observed full width at zero intensity (FWZI) of emission 


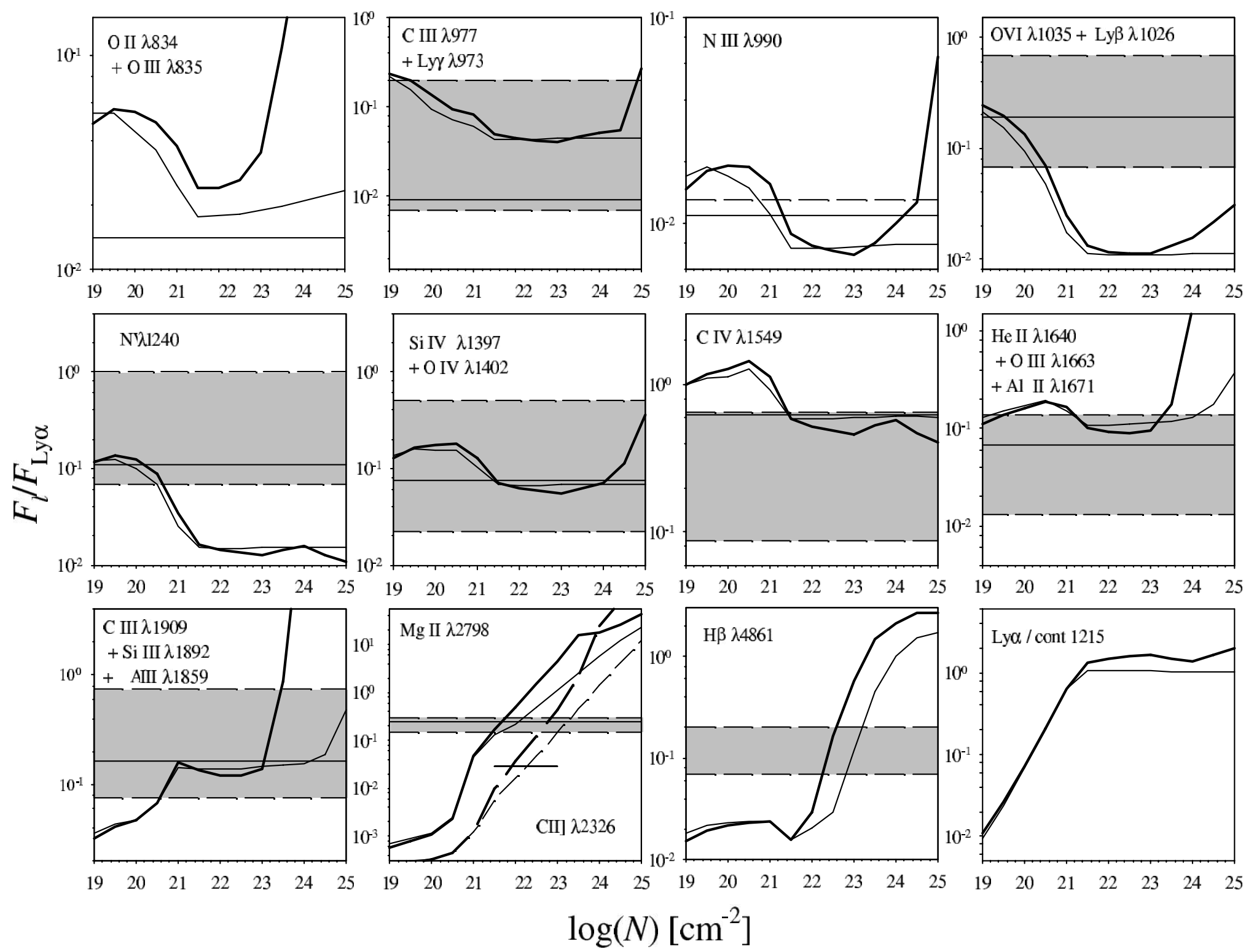

FIG. 2.- Lines and line blend ratios relative to Ly $\alpha$ as a function of $\log N$, where $N$ is the column density in $\mathrm{cm}^{-2}$. Thick solid curves show the calculations for $v=400 \mathrm{~km} \mathrm{~s} \mathrm{~s}^{-1}$, and thin solid curves show

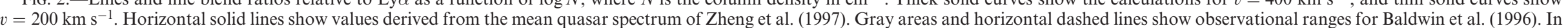

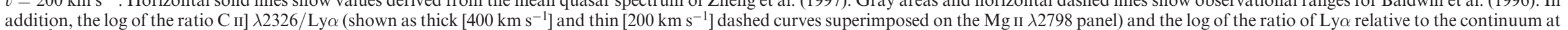
$1216 \AA$ (Ly $\alpha / \lambda 1216)$ are shown. 
lines $\left(V_{\mathrm{FWZI}} \sim 3 \times 10^{4} \mathrm{~km} \mathrm{~s}^{-1}\right.$; Peterson 1997) and by assuming rough equipartition of the macroscopic velocity field with gravity $\left(V_{\mathrm{FWHM}^{2}} \approx G M / R_{\text {in }}\right)$. This gives an inner radius of

$$
R_{18, \text { in }} \approx 1.5 \times 10^{-3} M_{8} .
$$

Note that for the estimated mass of the black hole in NGC 5548 ( $M_{8} \approx 0.7$; Peterson \& Wandel 1999) $R_{\text {in }} \approx 0.5$ lt-day. This is roughly consistent with the timescale for continuum variations (Dietrich et al. 2001). For $R<R_{\text {in }}$ dissipation may or may not exist. If it does, then it will produce continuum rather than line emission as a result of the high densities.

The outer edge of the BLR is taken to be the radius of dust sublimation because the production of dust reduces line emission. This occurs when $\Phi \approx 10^{18} \mathrm{~cm}^{-2} \mathrm{~s}^{-1}$ (Netzer $\&$ Laor 1993). The result is an outer radius of

$$
R_{18, \mathrm{BLR}} \approx 0.980\left(M_{8} \eta_{-1}\right)^{1 / 2} .
$$

At this radius the velocity of the turbulent field, according to equation (3), is $v_{3}\left(R_{18, \mathrm{BLR}}\right) \approx 0.025 L_{13}^{1 / 3} M_{8}^{1 / 6} \eta_{-1}^{-5 / 12}(25$ $\mathrm{km} \mathrm{s}^{-1}$ ). This is only a factor of a few larger than the thermal line width of BLR gas at $\sim 10^{4} \mathrm{~K}$ (Rees 1987).

\subsection{A Simple BLR Model: Results}

Observations suggest that the ionizing continuum is a factor of $\sim 2$ too weak to power the lines, given a reasonable covering factor (Korista et al. 1998). We hypothesize that this extra factor comes from turbulent heating. The logarithm of the dissipative luminosity (eq. [11]), relative to the Eddington luminosity, for various $\delta$ and $\gamma$ is shown in the contour plot of Figure 3. The calculations in the figure use $M_{8}=0.7$ and a covering factor of 0.1 . Values range from -4.0 in the upper right-hand corner to 1.0 in the lower lefthand corner of Figure 3. For the canonical covering factor $(0.1)$, values of dissipation rates in the upper right-hand corner are too low to provide the extra (noncontinuum) energy needed to explain the brightness of emission lines relative to the continuum.

Values of dissipation rates in the lower left-hand corner are too large. The result is emission lines far brighter than observed, exceeding the Eddington luminosity by a factor of $\sim 10$. This in itself is not physically impossible since the energy is going into turbulence rather than photons. A smaller covering factor would compensate for the enhanced luminosity and would further reduce the mass in BLR clouds. But if this were the case, the lines would be entirely powered by turbulence rather than photoionization.

AGN clouds show significant responses to temporal variations of the continuum. This suggests that the energy in the ionizing continuum must be only comparable to the dissipative heating, unless the dissipative heating rate is also modulated with the continuum. We note that in some cases emission-line profile variations are not correlated with changes in the continuum. One possible explanation is structural change in the distribution of the line-emitting gas in the BLR (Wanders \& Peterson 1996; Bottorff \& Ferland 2000). A complementary possibility is that dissipative heating in different regions of the BLR is being made manifest as additional line emission. Such variations may have an ana$\log$ in the ISM where dissipative heating is suggested to be the cause of intermittency in the kurtosis of ISM line profiles

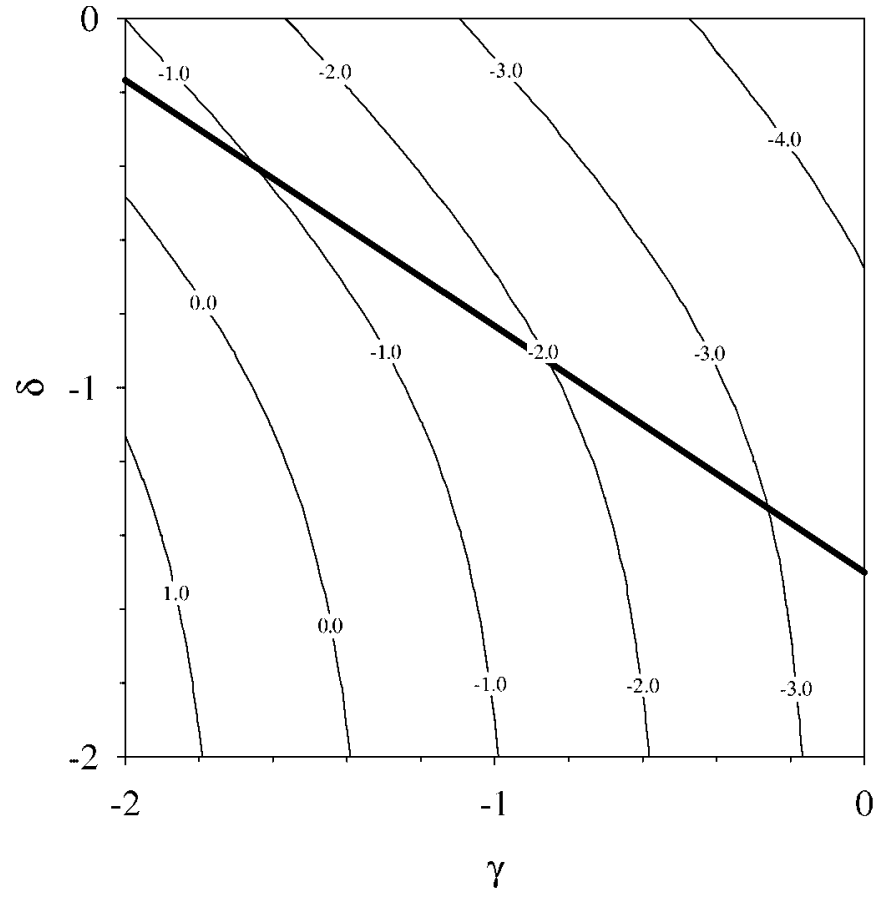

FIG. 3.-Contour plot showing the logarithm of the total power dissipated relative to the Eddington luminosity as a function of $\gamma$ and $\delta$ for the simple power-law model described in $\S 4.2$. Parameters held fixed in the calculation are the covering fraction $(0.1)$, the portion of the accretion efficiency that produces the continuum $(\eta=0.1)$, and the mass of the central black hole $\left(M_{8}=0.7\right)$. The thick line shows the pressure-law model described in Goad et al. (1993).

(Falgarone 1998). We note, however, that the overall time steady kurtosis of BLR emission lines is most likely dominated by systematic flows and/or beaming effects (Emmering et al. 1992).

Viable solutions, with modest luminosity and covering factor, lie between contour levels -1.0 and -2.0 . Along the contour level -2.0 the dissipative luminosity is about equal to the continuum luminosity intercepted by the BLR clouds. In this case the total line output will be twice the case in which there is no dissipative heating. This only requires an $\sim 10 \%$ increase in the mass accretion rate or efficiency. Large covering factors or beamed continua (Korista et al. 1997a) would not be required if dissipative heating is present.

The thick line in Figure 3 running through this region marks various pressure models for the BLR as outlined in Goad, O'Brien, \& Gondhalekar (1993). These were the parameters used in the analysis of emission-line ratios and reverberation responses in NGC 5548. The line traverses the regions between the -1.0 and -2.0 contours, the range where heating by photoionization and turbulence are competitive.

\section{CONCLUSIONS}

\subsection{Discussion}

The arguments presented above show that dissipative turbulence can account for the observed AGN emissionline spectrum for cloud parameters with $\log n=10.0$, $\log N \approx 22.0$, and $v \approx 200 \mathrm{~km} \mathrm{~s}^{-1}$. The standard and dis- 
sipative cloud models are directly compared in Table 1. The first four rows show the physical parameters. These are followed by the line ratios relative to $\operatorname{Ly} \alpha$ that we have discussed in $\S 4$. Following the computed lines is a pair of symbols consisting of "y," "n," or " _". The first corresponds to the Zheng et al. (1997) data and the second to the Baldwin et al. (1996) data. The symbol "y" signifies that the model agrees with the observations, " $\mathrm{n}$ " indicates that the model does not agree, and " ", occurs when the line is not included in the data set. The last row of the table shows a "Scorecard" of the number of consistent lines for the Zheng et al. (1997) and Baldwin et al. (1996) data sets relative to the total. It is clear that the standard and dissipative clouds have equal success. The dissipative cloud, however, is more consistent with the energetics and smooth line profiles.

A column density of $\log N \approx 22.0$ is 10 times smaller than the standard BLR cloud. For the same turbulence smaller clouds produce a smaller luminosity per cloud, but this does not necessarily mean that the total luminosity would be reduced. If equipartition (eq. [3]) prevails, $Q$ is independent of $L$ (substitute eq. [3] into eq. [2]). Thus, effects (if any) of smaller $L$ on the total luminosity are contained entirely within the covering factor (or equivalently the volume filling factor). There is only a small difference between the covering factor used for the extended models of Figure $3(0.1)$ and the value derived for the single-cloud model for $v=200 \mathrm{~km} \mathrm{~s}^{-1}$ in $\S 4(\sim 0.07)$. Thus, if the actual BLR is similar to the pressure-law models of Figure 3, the accretion rate or efficiency would need only to be increased by $\sim 10 \%$. To this must be added any kinetic luminosity that might be present as part of a systematic outflow, which could rival the continuum luminosity of the central engine (Bottorff \& Ferland 2001, § 5.4). We do not address how the turbulence is driven, which is beyond the scope of this paper, although $\S 2$ did show that turbulence is ubiquitous in nature. We conclude that the dissipation of turbulence into heat within BLR clouds of AGNs is able to explain why emission lines appear more luminous than expected for nonturbulent models and only requires a modest increase in the accretion rate or efficiency.

\subsection{Summary}

In this paper we investigated the effect that dissipative heating has on the production of bright emission lines and emission-line blends of quasars and other AGNs. We have analyzed line emission from a single BLR cloud model subject to varying degrees of turbulence, considered the effect that the column density has on line emission from clouds with dissipative turbulence, and estimated the luminosity required to drive this postulated dissipative heating. Although the theory of turbulent heating is complex and rich, with many details yet to be understood, a simple treatment does reveal the expected effects on a predominantly photoionized cloud. Details of the source of this turbulent heating do not greatly affect our results since we focus on the spectral signatures of this extra heating. As a result of our calculations we make the following conclusions:

1. Intensities of bright quasar emission lines increase with dissipative turbulent velocity. This is in accord with our previous findings for nondissipative turbulence (B00) but with the additional effect that internal cloud heating further enhances the production of low-ionization and collisional lines.

2. The predicted emission-line spectrum of a single-cloud model is in broad agreement with observations when $v \sim 200 \mathrm{~km} \mathrm{~s}^{-1}$. The low-ionization lines Mg II $\lambda 2798$ and $\mathrm{C}$ II] $\lambda 2326$ agree with observations for a column density of $N \sim 10^{22} \mathrm{~cm}^{-1}$. This dissipative cloud is as successful as a standard BLR cloud in reproducing the relative intensities of emission lines.

3. The dissipative BLR cloud model explains why emission lines are observed to be so smooth.

4. Simple estimates of the total luminosity in dissipative turbulence show that either the accretion rate or efficiency would need to be enhanced by $\sim 10 \%$ to account for the dissipative energy produced by this heating.

Nebular astrophysics research at the University of Kentucky is supported by NSF and NASA through grants AST 0071180 and NAG 5-8212. We would also like to thank Dinshaw Balsara, Jack Baldwin, and Kirk Korista for their careful reading of the manuscript.

\section{REFERENCES}

Arav, N., Barlow, T. A., Laor, A., \& Blandford, R. D. 1997, MNRAS, 288, 1015

Arav, N., Barlow, T. A., Laor, A., Sargent, W. L. W., \& Blandford, R. D. 1998, MNRAS, 297, 990

Baldwin, J. A., et al. 1996, ApJ, 461, 664

Baldwin, J. A., Ferland, G. J., Korista, K. T., \& Verner, D. 1995, ApJ, 455, L119

Balsara, D. S., Porquet, A., Ward-Thompson, D., \& Crutcher, R. M. 1999, in Proc. 2d Guillermo Haro Conf., Interstellar Turbulence, ed. J. Franco \& A. Carraminana (Cambridge: Cambridge Univ. Press), 261

Blandford, R. D. \& Payne, D. G. 1982, MNRAS, 199, 883

Bottorff, M. C., \& Ferland, G. J. 2000, MNRAS, 316, 103 2001, ApJ, 549, 118

Bottorff, M. C., Ferland, G. J., Baldwin, J., \& Korista, K. 2000a, ApJ, 542, 644 (B00)

Bottorff, M. C., Korista, K. T., \& Shlosman, I. 2000b, ApJ, 537, 134

Chelouche, D., \& Netzer, H. 2001, MNRAS, 326, 916

Choudhuri, A. R. 1998, The Physics of Fluids and Plasmas: An Introduction for Astrophysicists (Cambridge: Cambridge Univ. Press)

Collin-Souffrin, S. 1986, A\&A, 166, 115

Collin-Souffrin, S., \& Lasota, J.-P. 1988, PASP, 100, 1041

Corbin, M. R. 1990, ApJ, 357, 346

Crenshaw, D. M., \& Kraemer, S. B. 1999, ApJ, 521, 572

Crenshaw, D. M., Kraemer, S. B., Boggess, A., Maran, S. P., Mushotzky,

R.F. \& Wu, C. 1999 , ApJ, 516, 750

Crutcher, R. M. 1999, ApJ, 520, 706

Davidson, K., \& Netzer, H. 1979, Rev. Mod. Phys., 51, 715

Dietrich, M., et al. 2001, A\&A, 371, 79

Dietrich, M., Wagner, S. J., Courvoiser, T. J.-L., Bock, H., \& North, P. 1999, A\&A, 351, 31

Done, C., \& Krolik, J. H. 1996, ApJ, 463, 144

Dumont, A.-M., Collin-Souffrin, S., \& Nazarova, L. 1998, A\&A, 331, 11

Elmegreen, B. G. 1997, ApJ, 477, 196

Emmering, R. T., Blandford, R. D., \& Shlosman, I. 1992, ApJ, 385, 460

Espey, B. R., Carswell, R. F., Bailey, J. A., Smith, M. G., \& Ward, M. J. 1989, ApJ, 342, 666

Falgarone, E. 1998, in Proc. IAU Colloq. 166, Lecture Notes in Physics 506, The Local Bubble and Beyond, ed. D. Breitschwerdt, M. J. Freyberg, \& J. Truemper (Berlin: Springer), 251-260

Falgarone, E., \& Puget, J.-L. 1995, A\&A, 293, 840

Ferland, G. J. 1999, in ASP Conf. Ser. 162, Quasars and Cosmology, ed. G. Ferland \& J. Baldwin (San Francisco: ASP), 147

Ferland, G. J., Baldwin, J. A., Korista, K. T., Hamann, F., Carrswell, R. F., Phillips, M., Wilkes, B., \& Williams, R. E. 1996, ApJ, 461, 683

Frank, J., King, A. R., \& Raine, D. J. 1992, Accretion Power in Astrophysics (2d ed.; Cambridge: Cambridge Univ. Press)

Gaskell, C. M. 1982, ApJ, 263, 79

Goad, M. R., O'Brien, P. T., \& Gondhalekar, P. M. 1993, MNRAS, 263, 149

Goldstein, M. L., Ghosh, S., Siregar, E., \& Jayanti, V. 1999, in Lecture Notes in Physics 536, Nonlinear MHD Waves and Turbulence, ed. T. Passot \& P.-L. Sulem, (Berlin: Springer), 269 
Goldstein, M. L., Roberts, D. A., \& Matthaeus, W. H. 1995, ARA\&A, 33, 283

Goncalves, D. R., Friaca, A. C. S., \& Jantenco-Pereira, V. 2001, MNRAS, 328,409

Goncalves, D. R., Jantenco-Pereira, V., \& Opher, R. 1996, ApJ, 463, 489

Hamann, F., \& Ferland, G. J. 1999, ARA\&A, 37, 487

Kaspi, S., \& Netzer, H. 1999, ApJ, 524, 71

Königle, A., \& Kartje, J. F. 1994, ApJ, 434, 446

Korista, K. T., Baldwin, J. A., \& Ferland, G. J. 1998, ApJ, 507, 24

Korista, K. T., Baldwin, J. A., Ferland, G. J., \& Verner, D. 1997a, ApJS, 108,401

Korista, K. T., Ferland, G. J., \& Baldwin, J. A. 1997b, ApJ, 487, 555

Kwan, J., \& Carrol, T. J. 1982, ApJ, 261, 25

Kwan, J., \& Krolik, J. H. 1981, ApJ, 250, 478

Lucas, R., \& List, H. 1996, A\&A, 307, 237

Mac Low, M. 1999, ApJ, 524, 169

Mac Low, M.-M., Klessen, R. S., \& Burkert, A. 1998, Phys. Rev. Lett., 80, 2754

Mathur, S., Elvis, M., \& Wilkes, B. 1999, ApJ, 519, 605

Matthews, W. G. 1982, ApJ, 258, 425 1986, ApJ, 305, 187

Matthews, W. G., \& Doane, J. S. 1990, ApJ, 352, 423

Minter, A. H., \& Balser, D. S. 1997, ApJ, 484, L133

Minter, A. H., \& Spangler, S. R. 1997, ApJ, 485, 182

Murray, N., \& Chiang, J. 1998, ApJ, 494, 125

Murray, N., Chaing, J., Grossman, S. A., \& Voit, G. M. 1995, ApJ, 451, 498
Myers, P. C., \& Goodman, A. A. 1988, ApJ, 326, L27

Netzer, H. 1985, ApJ, 289, 451

Netzer, H., Brotherton, M. S., Wills, B. J., Han, M., Wills, D., Baldwin, J. A., Ferland, G. J., \& Brown, I. W. A. 1995, ApJ, 448, 27

Netzer, H., \& Laor, A. 1993, ApJ, 404, L51

Peterson, B. M. 1993, PASP, 105, 247

1997. An Introduction to Active Galactic Nuclei (Cambridge: Cambridge Univ. Press)

Peterson, B. M., et al. 1994, ApJ, 425, 622

Peterson, B. M., \& Wandel, A. 1999, ApJ, 521, L95

Porter, D. H., Pouquet, A., \& Woodward, P. R. 1994, Phys. Fluids, 6, 2133

Proga, D., Stone, J. M., \& Kallman, T. R. 2000, ApJ, 543, 686

Rees, M. J. 1987, MNRAS, 228, 47P

Shields, G. A. 1978, in Pittsburg Conf. on BL Lac Objects, ed. A. M. Wolfe (Pittsburgh: Univ. Pittsburgh Press), 257

Shlosman, I., Vitello, P., \& Shaviv, G. 1985, ApJ, 294, 96

Solomon, P. M., Rivolo, A. R., Barrett, J., \& Yahil, A. 1987, ApJ, 319, 730

Stone, J. M., Ostriker, E. C., \& Gammie, C. F. 1998, ApJ, 508, L99

Wanders, I., \& Peterson, B. M. 1996, ApJ, 466, 174

Weymann, R. J. 1997, in ASP Conf. Ser. 128, Mass Ejection from AGN, ed. N. Arav, I. Shlosman, \& R. J. Weymann (San Francisco: ASP), 3

Weymann, R. J., Carswell, R. F., \& Smith, M. G. 1981, ARA\&A, 19, 41

Wilkes, B. J. 1984, MNRAS, 207, 73

Zheng, W., Kriss, G. A., Telfer, R. C., Grimes, J. P., \& Davidsen, A. F. 1997, ApJ, 475, 469 